\title{
A multi-center survey on the postpartum mental health of mothers and attachment to their neonates during COVID-19 in Hubei Province of China
}

\author{
Sicong Peng ${ }^{1,2 \#}$, Yi Zhang ${ }^{1,2 \#}$, Hongyan Liu ${ }^{1,2 \#}$, Xiaona Huang ${ }^{3}$, Douglas James Noble ${ }^{3}$, Lixia Yang ${ }^{4}$, \\ Wei Lu ${ }^{5}$, Yahui Luo ${ }^{6}$, Huaping Zhu ${ }^{1,2}$, Li Cao ${ }^{1}$, Chunhua Liu ${ }^{1,7}$, Yang Chen ${ }^{1}$, Pei Zhang ${ }^{1}$, Shiwen Xia ${ }^{1,2}$, \\ Anuradha Narayan ${ }^{3}$
}

${ }^{1}$ Department of Neonatology, Maternal and Child Health Hospital of Hubei Province, Tongji Medical College, Huazhong University of Science and Technology, Wuhan, China; ${ }^{2}$ Clinical Research Center of the Neonatal Emergency Medicine of Hubei Province, Wuhan, China; ${ }^{3}$ Health, Nutrition and WASH, UNICEF China, Beijing, China; ${ }^{4}$ Department of Neonatology, Xiaochang First People's Hospital, Xiaochang, China; ${ }^{5}$ Department of Neonatology, Yichang Central People's Hospital, Yichang, China; ${ }^{6}$ Department of Pediatrics, Hanchuan People's Hospital, Hanchuan, China; ${ }^{7}$ Wuhan University of Science and Technology, Wuhan, China

Contributions: (I) Conception and design: S Xia, X Huang, A Narayan, DJ Noble; (II) Administrative support: S Xia, A Narayan; (III) Provision of study materials or patients: S Peng, Y Zhang, H Liu; (IV) Collection and assembly of data: S Peng, Y Zhang, H Liu; (V) Data analysis and interpretation: S Peng, X Huang, S Xia; (VI) Manuscript writing: All authors; (VII) Final approval of manuscript: All authors.

\#These authors contributed equally to this work.

Correspondence to: Shiwen Xia. Department of Neonatology, Maternal and Child Health Hospital of Hubei Province, Tongji Medical College, Huazhong University of Science and Technology, No. 745 Wuluo Road, Hongshan District, Wuhan 430070, China. Email: shiwenxia66@163.com; Anuradha Narayan. Health, Nutrition and WASH, UNICEF China, 12 Sanlitun Road, Chaoyang District, Beijing 100600, China. Email: anarayan@unicef.org.

Background: There is an emerging literature on the mental health of both pre- and post-partum mothers during the coronavirus disease 2019 (COVID-19) pandemic.

Methods: As of April 1, 2020, 23 mothers confirmed with COVID-19, 15 mothers suspected with COVID-19 but with negative polymerase chain reaction tests, and 33 mothers without COVID-19 (Control Group) were recruited for a study from Hubei Province in China. The Maternal Postnatal Attachment Scale (MPAS), the Zung Self-rating Anxiety Scale, and the Zung Self-rating Depression Scale were applied to investigate the attachment of mothers to their neonates and the postpartum mental health of mothers within the first 3 months after delivery (between 20 to 89 days).

Results: The period of mother-child separation among the confirmed group (33.9 \pm 20.9 days) was significantly longer than that of suspected group (16.7 \pm 12.2 days) and control group $(10.7 \pm 8.4$ days). The total score of the MPAS in mothers confirmed with COVID-19 $(45.5 \pm 4.2)$ was significantly lower (indicating less mother-child attachment) than that in the suspected $(50.5 \pm 4.7)$ and control (48.8 \pm 4.6$)$ groups. A negative correlation was noted between the mother-child separation time and the MPAS scores, including the subscale scores of attachment (MPAS acore: Spearman's $\rho=-0.33,95 \%$ CI: -0.095 to $-0.538, P=0.005$; Subscale score of attachment: Spearman's $\rho=-0.40,95 \%$ CI: -0.163 to $-0.592, \mathrm{P}=0.001)$. The incidence of postpartum anxiety in the confirmed, suspected and control groups was $4.3 \%, 6.7 \%$ and $12.1 \%$, respectively; and the incidence of postpartum depression was 39.1\%, 33.3\% and 30.3\%, respectively. No significant difference was found with regards to maternal postpartum anxiety and depression among the three groups.

Conclusions: Decreased mother-child attachment found among mothers confirmed with COVID-19, indicates that further intervention is needed to ensure mother-child interaction to appropriately develop attachment. Mother-child attachment experienced disruption due to prolonged mother-child separation necessitated by the COVID-19 management protocol, which needs to be revised to reduce prolonged mother-child separation. Additionally, mothers with and without COVID-19 suffered a high incidence of depression, which warrants further mental health investment for pregnant mothers during the COVID-19 pandemic. 
Keywords: COVID-19; postnatal; mental health; attachment

Submitted Aug 27, 2020. Accepted for publication Dec 07, 2020.

doi: 10.21037/atm-20-6115

View this article at: http://dx.doi.org/10.21037/atm-20-6115

\section{Introduction}

The coronavirus disease 2019 (COVID-19), caused by the novel coronavirus (SARS-CoV-2), is characterized as an acute and contagious respiratory disease (1). At present, the COVID-19 pandemic has spread to most countries and regions in the world, with 44.5 million cases recorded and more than 1.1 million deaths as of October 29, $2020(2,3)$. The Director General of the World Health Organization (WHO) on July 7, 2020 highlighted at a media briefing on COVID-19 that "the outbreak is accelerating and we have clearly not reached the peak of the pandemic" (2). Mental health challenges have been noted during the outbreak in China, with a higher prevalence of mental health problems reported among women in hardest-hit areas during the COVID-19 outbreak (4).

It has been reported that about $10-20 \%$ pregnant women and women in the early postpartum period suffer from mental health problems (5). Considering the risks of perinatal mental health morbidity can be increased under extreme stress (such as medical emergency, trauma and natural disasters), the mental health care of pregnant women should be urgently emphasized (6,7). Previous studies have investigated the effects of the COVID-19 pandemic on prenatal or postpartum depression and anxiety of women (8-10). The COVID-19 pandemic has also affected other types of maternal mental problems, such as fear, refusal to breastfeed, irritability, loneliness, and post-traumatic stress disorders (11). Additionally, the mother-child attachment is a potential advantage to early childhood development but little attention has been paid to it during the COVID-19 epidemic.

A multi-center longitudinal research study has been carried out in Hubei Province, the epicenter of the COVID-19 outbreak in China since February 10, 2020. In this study, we compared the postpartum mental health status between mothers confirmed or suspected with COVID-19 and mothers without COVID-19, and examined motherchild attachment within the first 3 months (between 20 to 89 days) after delivery. We present the following article in accordance with the STROBE reporting checklist (available at http://dx.doi.org/10.21037/atm-20-6115).

\section{Methods}

\section{Subjects}

Participants were recruited from Hubei Province for a multi-center longitudinal study on the transmission of COVID-19 and its impact on health and developmental outcomes among neonates born to mothers with confirmed/ suspected COVID-19. As of April 1, 2020, 71 pairs of pregnant women and their neonates from four hospitals have been recruited to this study (Figure 1). The participants of this study were from Wuhan (65 pairs), Xiaogan (four pairs), and Yichang (two pairs), three cities that ranked first, second and ninth, respectively, in terms of the number of confirmed COVID-19 cases in Hubei Province as of April 1, 2020. Among the participants, the confirmed group consisted of 23 pregnant women confirmed with COVID-19 and their neonates, including one set of twins; the suspected group included 15 pregnant women suspected of COVID-19 but found to be polymerase chain reaction (PCR) negative and their neonates; and the control group composed of 33 pregnant women who were not confirmed/suspected of COVID-19 and their neonates, including two sets of twins.

The Diagnosis and Treatment Scheme of Novel Coronavirus Pneumonia (Trial Seventh Edition) issued by Chinese National Health Commission was used for the diagnosis of COVID-19 (12). All the mothers in the confirmed group had a positive SARS-CoV-2 nucleic acid test using a throat swab or serological antibodies, with at least one clinical manifestation (e.g., fever, respiratory tract symptoms, chest CT of pneumonia, or abnormal blood routine) concurrently. The suspected group included mothers who had any epidemiological history within 14 days (e.g., travel or residence history in Wuhan and its surrounding areas or other communities with reported cases, close contact with COVID-19 confirmed cases), and at least two of the COVID-19 related clinical manifestations (e.g., fever, respiratory tract symptoms, chest CT of pneumonia), but subsequently had a negative SARS$\mathrm{CoV}-2$ nucleic acid test through throat swab and a negative serological test. In selecting the participants from the hospitals, mothers in confirmed and suspected groups were 


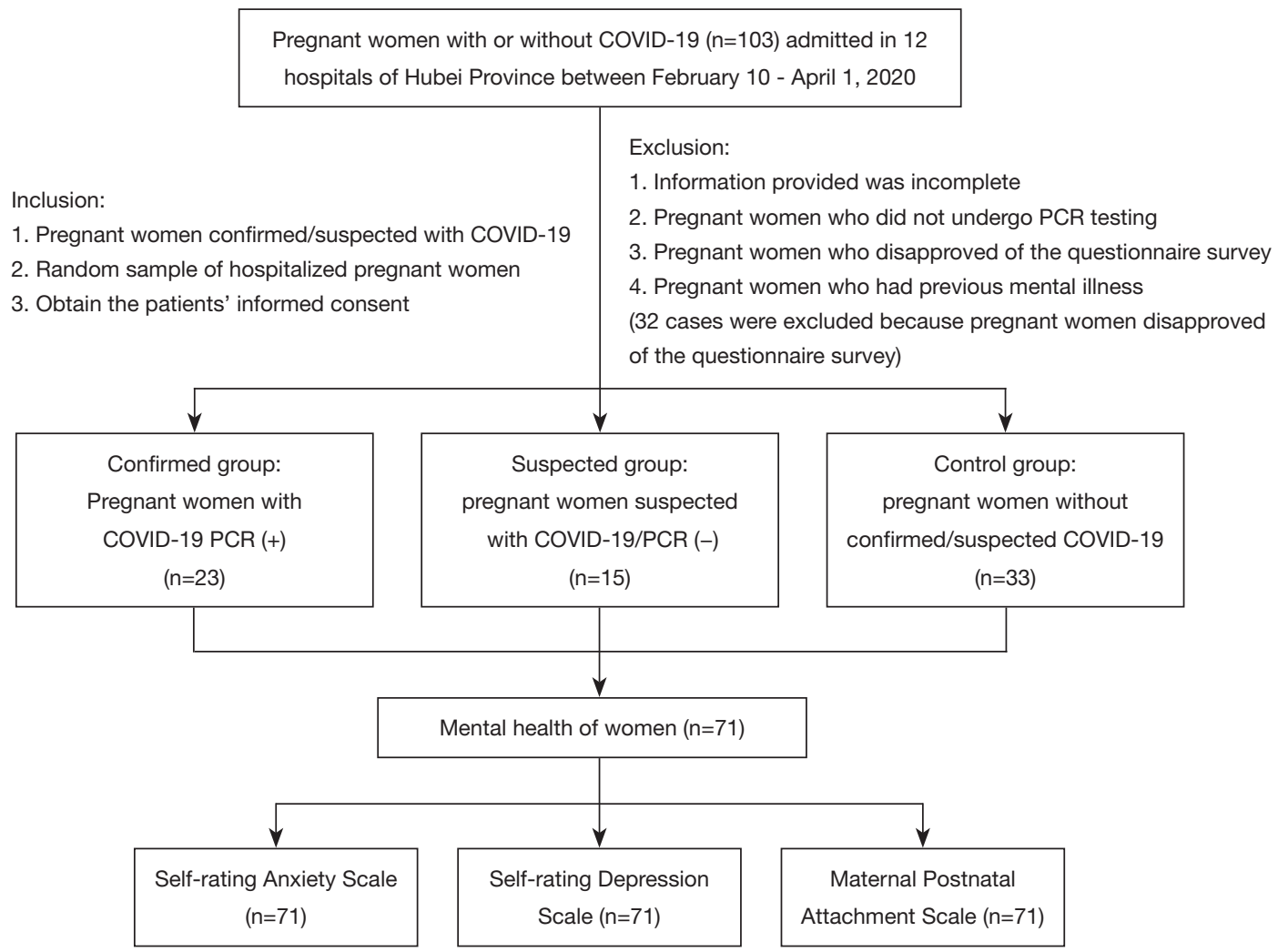

Figure 1 Research protocol and process of this study. The participants were enrolled between February 10-April 1, 2020, and the follow-up data were collected by May 15, 2020.

recruited from the available reported cases during February 10, 2020 to April 1, 2020. We used a random sampling method for the selection of mothers in the control group, from those who were admitted for birth or pregnancy complications in the same hospitals as the confirmed and suspected groups and matched by gestational age. These mothers were without COVID-19 symptoms and abnormal chest CT, and had a negative SARS-CoV-2 nucleic acid test, but presented with other perinatal diseases (e.g., premature delivery, gestational hypertension, gestational diabetes mellitus, gestational thyroid disease, fetal distress, premature rupture of membrane) or neonatal diseases (e.g., prematurity, hyperbilirubinemia, neonatal amniotic fluid/meconium aspiration syndrome, neonatal respiratory distress syndrome, neonatal bacterial pneumonia).

\section{Data collection and methods}

As of May 15, 2020, telephone-based follow-ups were conducted after delivery to collect information on motherchild attachment and the postpartum anxiety and depression of mothers. Mother-child attachment was assessed using the Maternal Postnatal Attachment Scale (MPAS) (13). Postpartum anxiety and depression of mothers were assessed by the Zung Self-rating Anxiety Scale (SAS) and the Zung Self-rating Depression Scale (SDS), respectively (14-16). Data were collected through the Questionnaire Star software within 3 months post-delivery, when the mothers were discharged from isolation and able to stay with their infants. Researchers familiar with the protocol conducted the telephone follow-ups, and were trained on the data collection tools and scales. The quality of data was checked by repeating the survey among half of the mothers randomly by telephone, and the results showed a high level of consistency.

The MPAS consists of two subscales on parenting anxiety and attachment respectively. A higher subscale score of parenting anxiety indicates more anxiety of the mothers when raising their children, and the higher subscale score of attachment means the mothers are more attached to their children (13). The cut off value was defined as 50 for the SAS and SDS; if the score was equal to or higher than 
the cut off value, mothers were suspected with postpartum anxiety or depression $(17,18)$. Commonly used for the diagnosis of anxiety and depressive disorders, the SAS and SDS have been validated in Chinese women and applied to postpartum women (16,19-21). Furthermore, mothers with SAS score of 50-59 were identified with mild anxiety, 60-69 with moderate anxiety, and $\geq 70$ with severe anxiety (17). Regarding depression, mothers with SDS score of 50-62 were identified with mild depression, 63-72 with moderate depression, and $\geq 73$ with severe depression $(17,18)$.

\section{Ethics}

This study has been registered in the Chinese Clinical Trial Register (ChiCTR2000033960). The study protocol was conducted in accordance with the Declaration of Helsinki (as revised in 2013) and has been approved by the Medical Ethics Committee of the Maternal and Child Health Hospital of Hubei Province in China (Approval Number: 2020-IEC-XM010). Informed consent was taken from all the participants before the study.

\section{Statistical analysis}

IBM SPSS Statistics software (version 22.0) was used for statistical analysis. The categorical variables were expressed as numbers and proportions (\%), and the Chi-square test or Fisher test was used to compare the proportions of the three groups, if applicable. The numerical variables were expressed as mean \pm standard deviation. One-way ANOVA was used to compare the data of the three groups which conformed to normality and homogeneity of variance. Kruskal-Wallis test was used to compare the numerical variables among the three groups which did not conform to the normality or homogeneity of variance. Unpaired Student's $t$ test or Mann-Whitney U test was used to compare the numerical variables between two groups which conformed or did not conform to the normality of variance, respectively. Spearman's correlation was used to assess the relationship between two numerical variables, which did not conform to the normality of variance. $P$ values less than 0.05 were considered as statistically significant.

\section{Results}

\section{Basic information of mothers}

Among the 23 mothers confirmed with COVID-19, their average age was 29.7 years old. Nearly $73.9 \%$ of them were primiparas (women giving birth for the first time), and $43.5 \%$ had a high school education or below. Two mothers had a positive PCR test result prior to delivery, and 21 mothers had positive results postnatally. All mothers confirmed with COVID-19 had abnormal chest imaging, and one of them had severe COVID-19 symptoms. Fifteen had fever or respiratory tract symptoms, while the remaining cases did not have typical COVID-19 symptoms (one had decreased amniotic fluid, two had premature rupture of membrane, and five were asymptomatic). Caesarean section was performed on $87.0 \%$ of the mothers, and one of the mothers had a twin pregnancy. The average gestational age of their neonates was 38.2 weeks (ranging 34-42 weeks), with an average birth weight of $3.0 \mathrm{~kg}$ (ranging $2.1-3.8 \mathrm{~kg}$ ). Among them, $52.2 \%$ had underlying pregnancy complications (including gestational hypertension, diabetes, thyroid disease, fetal distress, or intrauterine infection during pregnancy). None of their neonates had birth asphyxia.

Fifteen mothers in the suspected group and 33 mothers in the control group were enrolled in the study, and the average age was 32.6 and 30.2 years, respectively. Among the suspected and control groups, the percentages of pregnancy complications were $80.0 \%$ and $57.6 \%$, and caesarean section was performed on $66.7 \%$ and $48.5 \%$ of the mothers, respectively. The rate of caesarean section was significantly higher in the confirmed group than in the suspected and control groups ( $\mathrm{P}=0.01$, Chi-square test). There were no statistical differences between the three groups in age, educational level, incidence of pregnancy complications, or incidence of birth complications and abnormalities (e.g., amniotic fluid, umbilical cord and placenta abnormalities, fetal distress) (Table 1).

\section{Mother-child separation and attachment during daily interactions}

The maternal mental health survey was performed within 20 to 89 days, with an average length of about 2 months after delivery. There was no significant difference in the survey timing among the three groups (Table 2). Due to the COVID-19 pandemic, all babies and mothers in the three groups were separated after delivery and the length of separation lasted between 1 to 82 days due to hospitalization and/or centralized quarantine procedures. The babies in the control group were also separated from mothers due to other perinatal or neonatal diseases 
Table 1 Basic information of the mothers

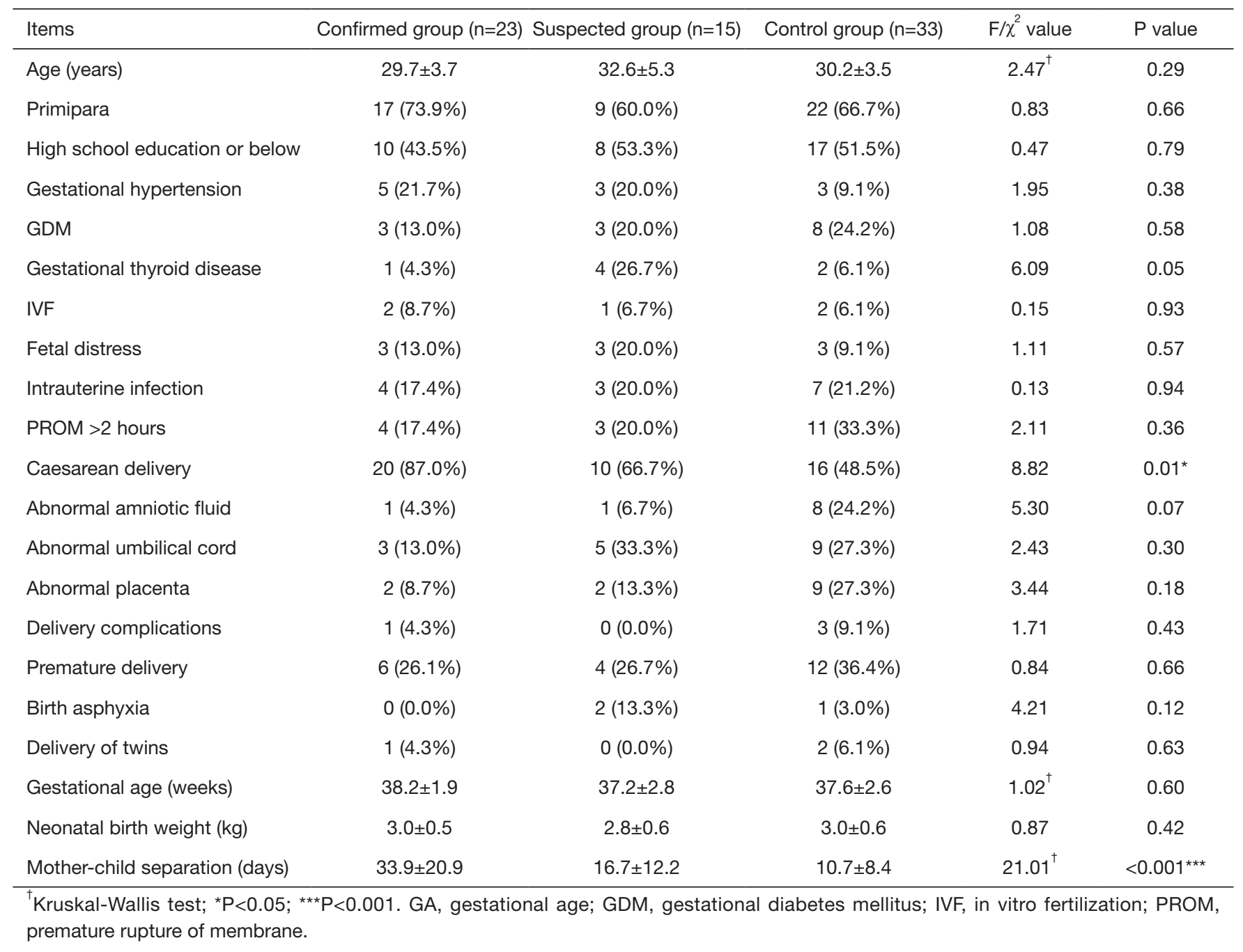

that needed hospitalization. The period of mother-child separation among the confirmed group $(33.9 \pm 20.9$ days $)$ was significantly longer than that of the other two groups (suspected group: $16.7 \pm 12.2$ days; control group: $10.7 \pm 8.4$ days; $\mathrm{P}<0.001$, Kruskal-Wallis test).

Mother-child attachment is a potential advantage to early childhood development, and could be influenced by mother-child separation, as well as maternal depression and anxiety (22-24). The average MPAS scores were 45.5, 50.5, and 48.8 for the confirmed, suspected and control groups, respectively, indicating the confirm group had significantly lower scores than the other two groups $(\mathrm{P}=0.003$, one-way ANOVA). The average subscale score of attachment in the confirmed group was $27.9 \pm 3.7$, which was significantly lower than the other two groups (suspected group: $32.3 \pm 4.4$; control group: $31.1 \pm 3.8 ; \mathrm{P}=0.002$, Kruskal-Wallis test). The average subscale scores of parenting anxiety among the confirmed, suspected and control groups were 17.6, 18.2 , and 17.8, respectively, without significant difference ( $\mathrm{P}=0.86$, one-way ANOVA).

Considering mother-child separation as a potential factor affecting the mother-child attachment, we analyzed the correlation between mother-child separation time and the MPAS scores (including the subscale scores). A negative correlation between the mother-child separation time and the MPAS scores was noted (Spearman's $\rho=-0.33,95 \%$ CI: -0.095 to $-0.538, \mathrm{P}=0.005)$. A similar significant negative correlation between the mother-child separation time and the subscale scores of attachment was also detected (Spearman's $\rho=-0.40,95 \%$ CI: -0.163 to $-0.592, \mathrm{P}=0.001$ ). 
Table 2 Questionnaire survey of maternal SAS, SDS and MPAS

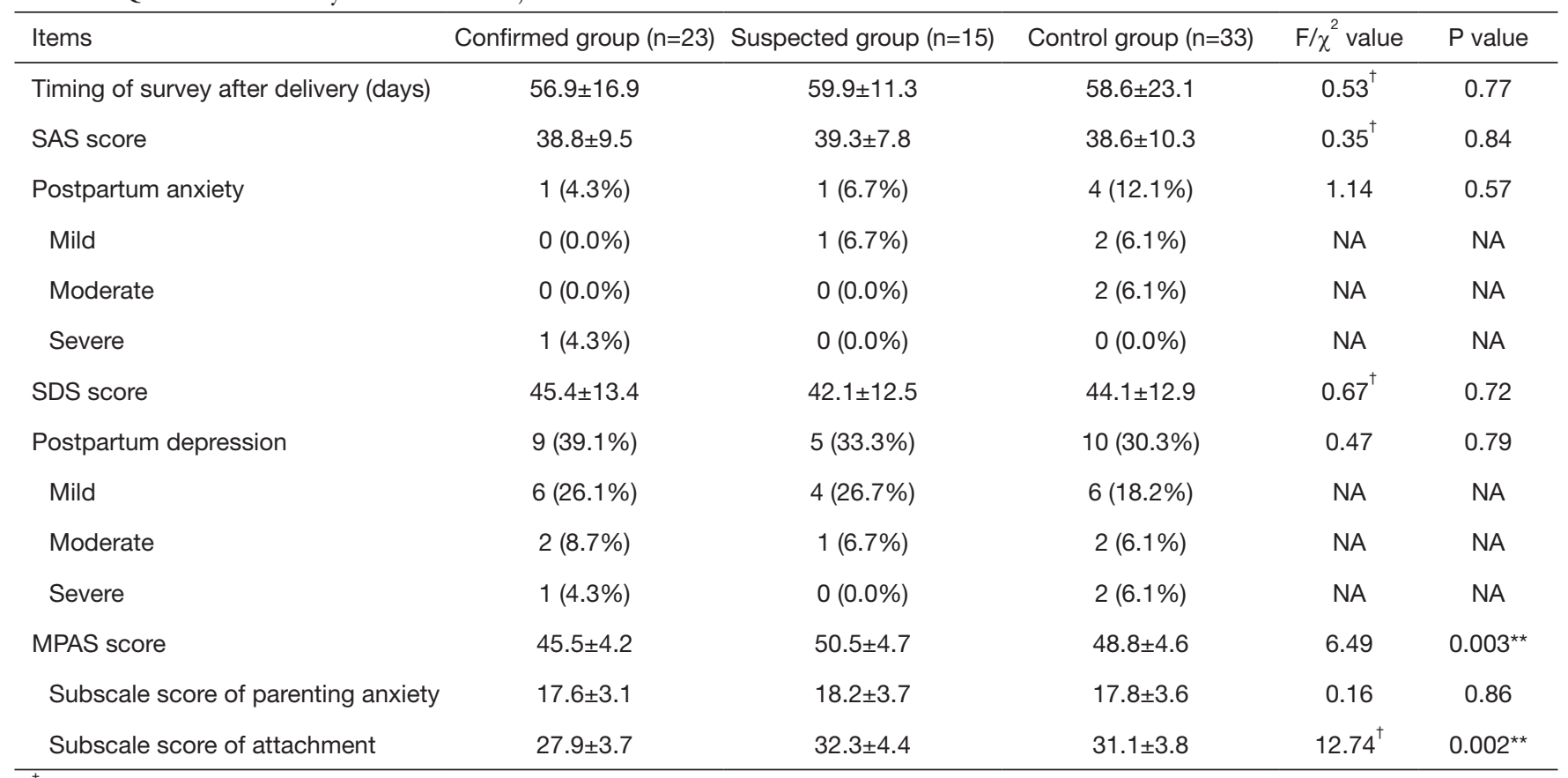

${ }^{\dagger}$ Kruskal-Wallis test; ${ }^{\star \star} \mathrm{P}<0.01$. SAS, Self-rating Anxiety Scale; SDS, Self-rating Depression Scale; MPAS, Maternal Postnatal Attachment Scale.

No significant correlation was found between the motherchild separation time and the subscale scores of parenting anxiety ( $\mathrm{P}=0.94$, Spearman's correlation).

\section{Postpartum anxiety and depression among mothers}

The incidence of postpartum anxiety among the confirmed, suspected and control groups was $4.3 \%, 6.7 \%$ and $12.1 \%$, respectively. The incidence of depression was $39.1 \%, 33.3 \%$ and $30.3 \%$, respectively (Table 2). Neither the SAS and SDS scores nor the incidence of anxiety and depression among the three groups showed significant difference (Table 2). Due to the small sample size, we did not perform additional statistical analysis among the subgroups of mild, moderate and severe postpartum anxiety or depression among the three groups.

The SAS and SDS scores both had no detectable correlation with the mother-child separation time $(\mathrm{P}=0.34$ for SAS and P=0.25 for SDS, Spearman's correlation). Additionally, the mother-child separation time between mothers with and without postpartum anxiety showed no significant difference (no anxiety group: $19.3 \pm 17.8$ days; anxiety group: $21.7 \pm 14.9$ days; $\mathrm{P}=0.34$, Mann-Whitney $\mathrm{U}$ test). The mothers with and without postpartum depression also showed the same pattern of mother-child separation (no depression group: $18.4 \pm 18.3$ days; depression group: $21.7 \pm 15.8$ days; $\mathrm{P}=0.25$, Mann-Whitney $\mathrm{U}$ test). Nonetheless, more mothers who were separated from their children for more than 14 days reported postpartum anxiety ( $\geq 14$ days: 4 of 35 cases, $11.4 \% ;<14$ days: 2 of 36 cases, $5.6 \% ; \mathrm{P}=0.43$, Fisher test) and depression ( $\geq 14$ days: 14 of 35 cases, $40.0 \%$; <14 days: 10 of 36 cases, $27.8 \%$; $\mathrm{P}=0.28$, Chi-square test) when compared with those who were separated from their children for less than 14 days. However, no statistical significance was detected, likely due the limited sample size.

Furthermore, we compared the MPAS scores and its subscale scores between mothers with and without anxiety or depression (Figure 2). It was found that the mothers with and without anxiety had similar MPAS scores (no anxiety group: $48.3 \pm 4.9$; anxiety group: $45.7 \pm 3.9 ; \mathrm{P}=0.20$, Unpaired Student's $t$ test, Figure $2 A$ ) and subscale scores of parenting anxiety (no anxiety group: $17.6 \pm 3.4$; anxiety group: $20.3 \pm 2.7$; $\mathrm{P}=0.06$, Unpaired Student's $t$ test, Figure $2 B$ ). However, the mothers with anxiety had remarkably lower subscale scores of attachment than mothers without anxiety (no anxiety group: $30.8 \pm 4.0$; anxiety group: $25.3 \pm 3.2 ; \mathrm{P}=0.005$, MannWhitney U test, Figure $2 C$ ). Additionally, the mothers 
A

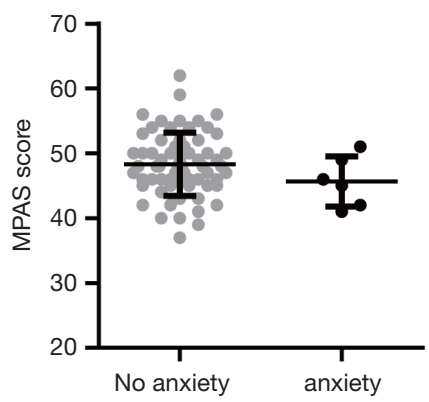

D

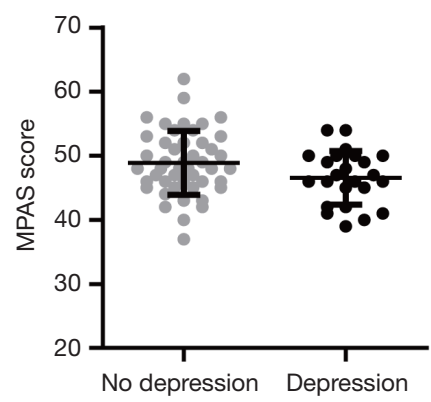

B

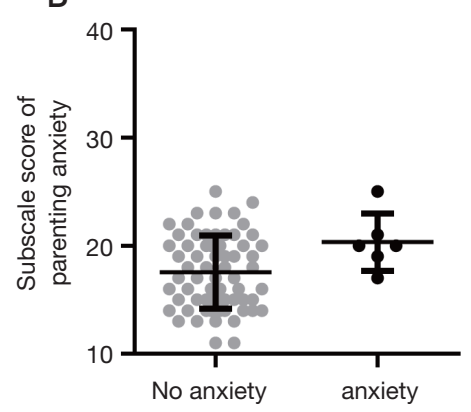

$\mathrm{E}$

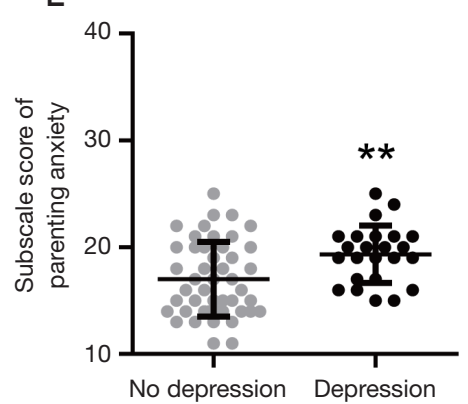

C

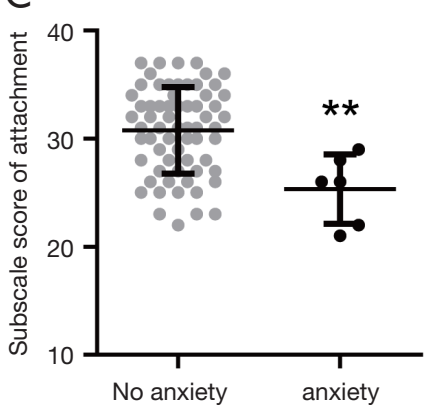

$\mathrm{F}$

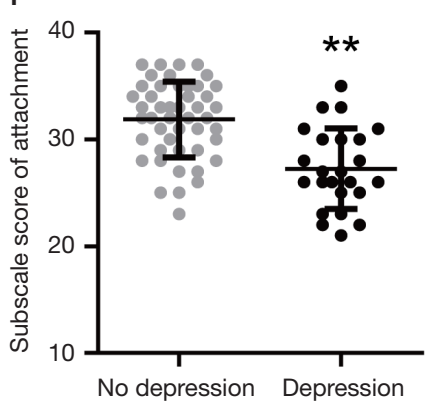

Figure 2 The MPAS scores as well as its subscale scores between the mothers with or without anxiety and depression. (A-C): The scatter plots showed that there was no statistical difference of the MPAS total scores (A) and the subscale scores of parenting anxiety (B) between mothers with or without postpartum anxiety. However, the subscale scores of attachment among mothers with postpartum anxiety were significantly lower than mothers without anxiety (C). (D,E,F): The scatter plots showed that there was no statistical difference of the MPAS total scores between mothers with or without postpartum depression (D). However, the subscale scores of parenting anxiety were significantly higher (E) while the subscale scores of attachment were significantly lower among mothers with postpartum depression (F). The black lines in the middle of scatter plots indicate the mean and the standard deviation. ${ }^{* *} \mathrm{P}<0.01$.

with and without depression also had similar MPAS scores (no depression group: 48.9 \pm 5.0 ; depression group: 46.6 $\pm 4.2 ; \mathrm{P}=0.06$, Unpaired Student's $t$ test, Figure $2 D$ ). But the mothers with depression presented increased subscale scores of parenting anxiety (no depression group: 17.0 \pm 3.5 ; depression group: $19.3 \pm 2.7 ; \mathrm{P}=0.003$, Unpaired Student's $t$ test, Figure $2 E$ ) and decreased subscale scores of attachment (no depression group: 31.9 \pm 3.5 ; depression group: $27.3 \pm 3.8 ; \mathrm{P}<0.001$, Mann-Whitney $\mathrm{U}$ test, Figure $2 F$ ) compared to mothers without depression. These results indicated that both postpartum anxiety and depression had negative impacts on the mother-child attachment.

\section{Discussion}

\section{Postpartum mother-child attachment during the COVID-19 pandemic}

Maternal mental health is associated with short-term and long-term risks for the mothers' health and wellbeing, as well as their children's physical, cognitive and psychological development (7). Maternal depression threatens mothers' emotional and physical capacity to care for and foster a healthy relationship with their children (22). Furthermore, it has been reported that depressive symptoms are negatively associated with mothers' emotional bonding to their children (23). The MPAS is an international scale to evaluate mother-child attachment, and has been adapted for use in China with both validity and reliability tested and verified (13). In our study, we found that the mothers in the confirmed group had less attachment to their children when compared with the suspected and control groups. However, whether the decrease in mother-child attachment will persist and ultimately influence the development of children still needs further research. Considering that motherchild attachment is the basis for children to establish their own attachment system, which is related to children's 
social, emotional and cognitive development $(13,25)$, it is important to provide support that allows for secure motherchild attachment and interaction, particularly for families with mothers confirmed with COVID-19.

\section{Impact of mother-child separation on the mother-child attachment}

During the COVID-19 epidemic in China, many expecting mothers with or without COVID-19 suffered from motherchild separation due to hospitalization and centralized quarantine procedures. Chinese national guidelines for COVID-19 required an additional 14 days of isolation for confirmed/suspected patients after they are discharged from the hospital, even for mothers suspected of COVID-19 who had negative test results (12). These measures likely resulted in prolonged mother-child separation and negative consequences on maternal mental health (24). In our study, the mother-child separation time among mothers with confirmed COVID-19 was 4-82 days, longer than that in the suspected (5-47 days) and control (1-34 days) groups. Previous studies have highlighted that mothers who are separated from their infants experienced acute suffering or severe stress, and these effects lasted for a long time $(24,26)$. Thus, it is particularly important to revisit the recommendations on isolation for expecting mothers, and develop better solutions to avoid unnecessary and prolonged mother-child separation.

\section{Postpartum anxiety and depression among mothers during COVID-19 pandemic}

Postpartum anxiety and depressive symptoms have been found to be associated with breastfeeding, mother-child emotional bonding and interaction, which may affect the health and development of infants $(23,27)$. During the COVID-19 outbreak, a variety of factors may have influenced the postnatal mental health of mothers with COVID-19, such as long-term home quarantine, infections during pregnancy, and prolonged mother-child separation. In this study, $8.5 \%$ (6 of 71 cases) and 33.8\% (24 of 71 cases) of mothers were detected with postpartum anxiety and depression across the three groups, respectively, and five mothers had both postpartum anxiety and depression. It seems there was no significant difference in the incidence of maternal postpartum depression and anxiety 2 months after giving birth between mothers confirmed or suspected with COVID-19 and mothers without COVID-19 in this study.
This may be because the sample size is not large enough to detect the relevant differences. Moreover, the control group in this study were not completely healthy mothers, as they had other perinatal complications during and after delivery, and thus experienced mother-child separation, which may have increased depression and anxiety in the control group, and reduced the difference between the three groups as a result. In addition, the mothers in the three groups were all from Hubei Province, and subject to the similar prevention and control measures, including lockdown of cities and home quarantine. Thus, the impact caused by the pandemic might be similar across the groups in terms of mental stress. Notably, the rate of postpartum anxiety in this study was lower than other reported studies (35.4-83.3\%) $(8,28)$, perhaps due to different measurements and the decrease of SAS scores at 4-6 weeks postpartum (19). A previous study reported that the incidence of maternal depressive symptoms evaluated by the Edinburgh Postnatal Depression Scale (EPDS) was about $26 \%$ before the COVID-19 epidemic in China, which increased to $29.6 \%$ after the declaration of the COVID-19 epidemic (9). Despite the fact that no significant difference was detected among the three groups in this study, the overall high incidence of depression across all three groups warrants special attention, particularly to maternal postpartum mental health during the COVID-19 pandemic, and necessary investment, services and support required.

The major limitation of this study was that the sample size for each group was relatively smaller compared to previous epidemiological studies $(9,29)$. This may limit the statistical detection of differences between the three groups, which should be taken into consideration when interpreting the findings. In addition, all the mothers in the study experienced the same context of COVID-19, it is therefore not possible to distinguish the maternal postpartum anxiety and depression that was specifically related to COVID-19.

\section{Conclusions}

In summary, mothers with or without COVID-19 in this study all suffered a high incidence of depression, which warrants investment and attention to improve mental health care and support. Additionally, decreased mother-child attachment among mothers confirmed with COVID-19 indicates that support for mother-child contact and interaction for ensuring appropriate attachment is urgently needed during the COVID-19 pandemic. Furthermore, mother-child attachment experienced disruption due 
to prolonged mother-child separation because of the COVID-19 management protocol, which needs to be revisited and improved to avoid unnecessary and prolonged mother-child separation.

\section{Acknowledgments}

Technical support: We thank professor Gong Yuhua for providing the Chinese version of the MPAS for the study.

Funding: The study was funded by the Hong Kong Committee for UNICEF (to SX).

\section{Footnote}

Reporting Checklist: The authors have completed the STROBE reporting checklist. Available at http://dx.doi. org/10.21037/atm-20-6115

Data Sharing Statement: The data supporting the conclusions of this article can be made available from the corresponding author upon request. Available at http:// dx.doi.org/10.21037/atm-20-6115

Conflicts of Interest: All authors have completed the ICMJE uniform disclosure form (available at http://dx.doi. org/10.21037/atm-20-6115). The authors have no conflicts of interest to declare. The opinions expressed in this paper are solely those of the authors and do not necessarily represent the official position of UNICEF.

Ethical Statement: The authors are accountable for all aspects of the work in ensuring that questions related to the accuracy or integrity of any part of the work are appropriately investigated and resolved. This study has been registered in the Chinese Clinical Trial Register (ChiCTR2000033960). The study protocol was conducted in accordance with the Declaration of Helsinki (as revised in 2013) and has been approved by the Medical Ethics Committee of the Maternal and Child Health Hospital of Hubei Province in China (Approval Number: 2020 IEC-XM010). Informed consent was taken from all the participants before the study.

Open Access Statement: This is an Open Access article distributed in accordance with the Creative Commons Attribution-NonCommercial-NoDerivs 4.0 International License (CC BY-NC-ND 4.0), which permits the noncommercial replication and distribution of the article with the strict proviso that no changes or edits are made and the original work is properly cited (including links to both the formal publication through the relevant DOI and the license). See: https://creativecommons.org/licenses/by-nc-nd/4.0/.

\section{References}

1. Huang C, Wang Y, Li X, et al. Clinical features of patients infected with 2019 novel coronavirus in Wuhan, China. Lancet 2020;395:497-506.

2. World Health Organization. WHO Director-General's opening remarks at the media briefing on COVID-19 - 7 July 2020 [cited 2020 Aug 24]. Available online: https:// www.who.int/dg/speeches/detail/who-director-general-sopening-remarks-at-the-media-briefing-on-covid-19---7july-2020

3. COVID-19 Dashboard by the Center for Systems Science and Engineering (CSSE) at Johns Hopkins University (JHU) 2020 [cited 2020 Oct 29]. Available online: https:// coronavirus.jhu.edu/map.html.

4. Liu N, Zhang F, Wei C, et al. Prevalence and predictors of PTSS during COVID-19 outbreak in China hardesthit areas: Gender differences matter. Psychiatry Res 2020;287:112921.

5. National Institute for Health and Care Excellence. Antenatal and postnatal mental health: clinical management and service guidance 2020 [cited 2020 Oct 29]. Available online: https://www.nice.org.uk/guidance/ cg192

6. Zeng LN, Chen LG, Yang CM, et al. Mental health care for pregnant women in the COVID-19 outbreak is urgently needed. Women Birth 2020; S1871-5192:302109. doi: 10.1016/j.wombi.2020.03.009.

7. Thapa SB, Mainali A, Schwank SE, et al. Maternal mental health in the time of the COVID-19 pandemic. Acta Obstet Gynecol Scand 2020;99:817-8.

8. Durankuş F, Aksu E. Effects of the COVID-19 pandemic on anxiety and depressive symptoms in pregnant women: a preliminary study. J Matern Fetal Neonatal Med 2020;18:1-7.

9. Wu Y, Zhang C, Liu H, et al. Perinatal depressive and anxiety symptoms of pregnant women along with COVID-19 outbreak in China. Am J Obstet Gynecol 2020;223:240.e1-9.

10. Ceulemans M, Hompes T, Foulon V. Mental health status of pregnant and breastfeeding women during the COVID-19 pandemic: A call for action. Int J Gynaecol Obstet 2020. Available online: https://pubmed.ncbi.nlm. 


\section{Page 10 of 10}

nih.gov/32620037. Epub $2020 \mathrm{Jul} 3$.

11. Vieira LG, Camargo ELS, Schneider G, et al.

Repercussions of the COVID-19 Pandemic on the Mental Health of Pregnant and Puerperal Women: A Systematic Review. medRxiv 2020. Available online: https://www. medrxiv.org/content/10.1101/2020.08.17.20176560v1. Epub 2020 Aug 21.

12. National Health Commission of the People's Republic of China. The Diagnosis and Treatment Scheme of Novel Coronavirus Pneumonia (Trial Seventh Edition) 2020 [cited 2020 Aug 24]. Available online: http://www.nhc.gov. cn/yzygj/s7653p/202003/46c9294a7dfe4cef80dc7f5912 eb1989.shtml.

13. Ge N, Hongxia F, Zhichun Z. Validity and Reliability of the Chinese Version of the Maternal Postnatal Attachment Scale. Chinese Journal of Clinical Psychology 2018;26:918-21.

14. Ma X, Wang $\mathrm{Y}, \mathrm{Hu} \mathrm{H}$, et al. The impact of resilience on prenatal anxiety and depression among pregnant women in Shanghai. J Affect Disord 2019;250:57-64.

15. Rolle L, Giordano M, Santoniccolo F, et al. Prenatal Attachment and Perinatal Depression: A Systematic Review. Int J Environ Res Public Health 2020;17:2644.

16. Liang $Y$, Chen $M$, Zheng $X$, et al. Screening for Chinese medical staff mental health by SDS and SAS during the outbreak of COVID-19. J Psychosom Res 2020;133:110102.

17. Ma Q, Su K, Fu Z, et al. Anxiety and depression in patients with nasal septal deviation. Am J Otolaryngol 2020;41:102450.

18. Peng M, Mo B, Liu Y, et al. Prevalence, risk factors and clinical correlates of depression in quarantined population during the COVID-19 outbreak. J Affect Disord 2020;275:119-24.

19. Liou SR, Wang P, Cheng CY. Longitudinal study of perinatal maternal stress, depressive symptoms and anxiety. Midwifery 2014;30:795-801.

20. Meades R, Ayers S. Anxiety measures validated in perinatal populations: a systematic review. J Affect Disord

Cite this article as: Peng S, Zhang Y, Liu H, Huang X, Noble DJ, Yang L, Lu W, Luo Y, Zhu H, Cao L, Liu C, Chen Y, Zhang P, Xia S, Narayan A. A multi-center survey on the postpartum mental health of mothers and attachment to their neonates during COVID-19 in Hubei Province of China. Ann Transl Med 2021;9(5):382. doi: 10.21037/atm-20-6115
Peng et al. Postpartum mental health of mothers with COVID-19

2011;133:1-15.

21. Lei M, Li C, Xiao X, et al. Evaluation of the psychometric properties of the Chinese version of the Resilience Scale in Wenchuan earthquake survivors. Compr Psychiatry 2012;53:616-22.

22. Lefkovics E, Baji I, Rigo J. Impact of maternal depression on pregnancies and on early attachment. Infant Ment Health J 2014;35:354-65.

23. Edhborg M, Nasreen HE, Kabir ZN. Impact of postpartum depressive and anxiety symptoms on mothers' emotional tie to their infants 2-3 months postpartum: a population-based study from rural Bangladesh. Arch Womens Ment Health 2011;14:307-16.

24. Tomori C, Gribble K, Palmquist AEL, et al. When separation is not the answer: Breastfeeding mothers and infants affected by COVID-19. Matern Child Nutr 2020;16:e13033.

25. Maddahi MS, Dolatian M, Khoramabadi M, et al. Correlation of maternal-fetal attachment and health practices during pregnancy with neonatal outcomes. Electron Physician 2016;8:2639-44.

26. Palmquist AEL, Holdren SM, Fair CD. "It was all taken away": Lactation, embodiment, and resistance among mothers caring for their very-low-birth-weight infants in the neonatal intensive care unit. Soc Sci Med 2020;244:112648.

27. Ritchie-Ewing G, Mitchell AM, Christian LM. Associations of Maternal Beliefs and Distress in Pregnancy and Postpartum with Breastfeeding Initiation and Early Cessation. J Hum Lact 2019;35:49-58.

28. Corbett GA, Milne SJ, Hehir MP, et al. Health anxiety and behavioural changes of pregnant women during the COVID-19 pandemic. Eur J Obstet Gynecol Reprod Biol 2020;249:96-7.

29. Zanardo V, Manghina V, Giliberti L, et al. Psychological impact of COVID-19 quarantine measures in northeastern Italy on mothers in the immediate postpartum period. Int J Gynaecol Obstet 2020;150:184-8. 